\title{
MicroRNA-421 participates in vitiligo development through regulating human melanocyte survival by targeting receptor-interacting serine/threonine kinase 1
}

\author{
XUECHENG SUN* ${ }^{*}$ TAO WANG* ${ }^{*}$ BO HUANG, GAOBO RUAN and AIE XU \\ Department of Dermatology, The Third People's Hospital of Hangzhou, Hangzhou, Zhejiang 310009, P.R. China
}

Received June 18, 2019; Accepted November 22, 2019

DOI: $10.3892 / \mathrm{mmr} .2019 .10878$

\begin{abstract}
Vitiligo is a common localized or generalized skin pigmentation disorder. Endoplasmic reticulum (ER) stress may be implicated in the development of vitiligo. microRNA-421 (miR-421) has been reported to be dysregulated in various human tumors. However, there is no report to date on the role of miR-421 in vitiligo development. The present study demonstrated that $3 \mu \mathrm{M}$ tunicamycin (TM) increased the expression of the ER stress-related proteins protein kinase RNA-like endoplasmic reticulum kinase (PERK), $\alpha$ subunit of eukaryotic translation initiation factor 2 (eIF2 $\alpha$ ) and C/EBP homologous protein (CHOP) in human primary epidermal melanocytes. Moreover, TM suppressed melanocyte viability and induced apoptosis. Reverse transcription-quantitative PCR analysis demonstrated that TM promoted miR-421 expression in human melanocytes. Next, TargetScan and dual luciferase reporter gene assay indicated that receptor-interacting serine/threonine kinase 1 (RIPK1) was a direct target of miR-421. RIPK1 expression was significantly downregulated in TM-induced human melanocytes. Subsequently, the effect of miR-421 downregulation on the damage of human melanocytes induced by ER stress was investigated. Human melanocytes were transfected with inhibitor control, miR-421 inhibitor, miR-421 inhibitor + control-short hairpin (sh)RNA, or miR-421 inhibitor + RIPK1-shRNA for $24 \mathrm{~h}$ and then treated with TM $(3 \mu \mathrm{M})$ for $48 \mathrm{~h}$. TM was found to upregulate PERK, eIF $2 \alpha$ and CHOP protein expression in human melanocytes, which was reduced by an miR-421 inhibitor. In addition, the miR-421 inhibitor increased viability and reduced apoptosis in TM-treated melanocytes. Furthermore, all these effects of
\end{abstract}

Correspondence to: Dr Aie Xu, Department of Dermatology, The Third People's Hospital of Hangzhou, 38 Xihu Road, Hangzhou, Zhejiang 310009, P.R. China

E-mail: xa20191806@163.com

\section{${ }^{*}$ Contributed equally}

Key words: microRNA-421, vitiligo, receptor-interacting serine/ threonine kinase 1, human melanocytes, endoplasmic reticulum stress the miR-421 inhibitor on TM-induced human melanocytes were reversed by RIPK1-shRNA. Further analyses revealed that the miR-421 inhibitor activated the phosphoinositide 3 kinase/protein kinase $\mathrm{B} /$ mammalian target of rapamycin signaling pathway in TM-induced human melanocytes. These data collectively suggest that miR-421 may serve as a new treatment target in vitiligo development.

\section{Introduction}

Vitiligo is a common idiopathic disease that is characterized by the destruction of melanocytes $(1,2)$. Although the prevalence of vitiligo is $<1 \%$ globally, it may be as high as $3 \%$ in some populations (2). At present, patients with vitiligo have no other symptoms except for skin discoloration, but the quality of life of some patients may be severely compromised $(3,4)$. Vitiligo is an autoimmune disease and it has been reported that multiple immune response genes may be involved in its development (5). Previous studies have demonstrated that vitiligo may be caused by T-cell-mediated oxidative stress and may involve certain mediators such as tumor necrosis factor (TNF)- $\alpha$, heat shock protein 70 and interleukin-1 $\beta$ (6-8). The destruction of melanocytes is caused by the imbalance of reactive oxygen species, which leads to damage of skin melanocytes by free radicals, leading to structural damage of proteins, apoptosis, activation of cytokines and endoplasmic reticulum (ER) damage (6-8). The ER is an important organelle, which is mainly responsible for protein biosynthesis and folding. ER stress is characterized by accumulation and aggregation of unfolded and/or misfolded proteins in the ER lumen (9).

MicroRNAs (miRNAs/miRs) are small and highly conserved regulatory RNA molecules $\sim 22$ nucleotides in length (10). miRNAs can regulate gene expression at the post-transcriptional level by targeting their 3'-untranslated region (UTR) to promote degradation or inhibit translation of mRNA (11). Accumulating evidence has demonstrated that miRNAs participate in the development and progression of various human cancers (12-14). The expression of miR-421 was found to be abnormal in several types of cancer. A previous study demonstrated that miR-421 acted as a tumor promoter in pancreatic cancer through targeting DPC4/mothers against decapentaplegic homolog 4 (15). Wu et al (16) reported that miR-421 was significantly upregulated in human gastric 
cancer tissues and promoted proliferation of gastric cancer cells by downregulating caspase-3 expression. However, the role of miR-421 in vitiligo patients is unclear.

Receptor-interacting serine/threonine kinase 1 (RIPK1) is a crucial regulator of TNF receptor 1 (TNFR1) signaling (17). RIPK1 plays a major role in the pathogenesis and prognosis of liver diseases $(18,19)$. Previous research demonstrated that RIPK1-mediated necrotic apoptosis may also occur in neurons, leading to the development of neurodegenerative diseases (20). However, the expression and role of RIPK1 in vitiligo patients remain unclear.

The phosphoinositide 3-kinase (PI3K)/protein kinase B (AKT)/mammalian target of rapamycin (mTOR) pathway has been found to be associated with cell survival in response to oxidative stress (21). Growth factors may protect against oxidative stress-induced apoptosis through activation of the AKT and mTOR pathways (22-24). In addition, indirect data indicated that $\alpha$-melanocyte-stimulating hormone ( $\mathrm{MSH}$ ) stimulates melanogenesis through the activation of MEK/extracellular signal-regulated kinase (ERK) or PI3K/AKT (25). Modulation of the PI3K/AKT/mTOR signaling pathway may be a novel approach to the clinical management of vitiligo (26). However, the association between miR-421 and the PI3K/AKT/mTOR pathway in melanocytes under ER stress remains unclear.

The aim of the present study was to determine the role of miR-421 in vitiligo development and to explore the underlying mechanism.

\section{Materials and methods}

Cell culture and transfection. Primary epidermal melanocytes were obtained from the American Type Culture Collection (cat no. ATCC ${ }^{\circledR}$ PCS-200-013) and cultured in Medium 254 (Gibco; Thermo Fisher Scientific, Inc.) supplemented with human melanocyte growth supplement (Gibco; Thermo Fisher Scientific, Inc.) at $37^{\circ} \mathrm{C}$ with $5 \% \mathrm{CO}_{2}$. Primary epidermal melanocytes $\left(1 \times 10^{6}\right.$ cells per well) were transfected with the inhibitor control (5'-CAGUACUUUUGUGUAGUACAA-3'; Guangzhou Ribobio Co., Ltd.), miR-421 inhibitor (5'-GCG CCCAAUUAAUGUCUGUUGAU-3'; Guangzhou Ribobio Co., Ltd.), $0.2 \mu \mathrm{M}$ control-shRNA (cat no. sc-108060; Santa Cruz Biotechnology, Inc.), $0.2 \mu \mathrm{M}$ RIPK1-shRNA (cat no. sc-44326-SH; Santa Cruz Biotechnology, Inc.), miR-421 inhibitor + control-shRNA, or miR-421 inhibitor + RIPK1-shRNA for $24 \mathrm{~h}$ using Lipofectamine 2000 reagent (Invitrogen; Thermo Fisher Scientific, Inc.) according to the manufacturer's protocol. The transfection efficiency was determined by reverse transcription-quantitative PCR (RT-qPCR) $24 \mathrm{~h}$ after cell transfection.

ER stress induction. To establish ER stress in human melanocytes, the cells were treated with $3 \mu \mathrm{M}$ tunicamycin (TM; Sigma-Aldrich; Merck KGaA) for $48 \mathrm{~h}$ according to a previous study (9).

$R T$-qPCR analysis. Total RNA was extracted from cells using TRIzol reagent (Invitrogen; Thermo Fisher Scientific Inc.) and stored at $-80^{\circ} \mathrm{C}$. Subsequently, total RNA was reverse transcribed into complementary DNA using a reverse transcription kit (Vazyme) according to the manufacturer's protocol. RT-qPCR was carried out by SYBR Green PCR Master Mix (Vazyme) following the manufacturer's protocols. U6 or GAPDH was used for normalization. The primer sequences used were as follows: U6, forward 5'-GCTTCG GCAGCACATATACTAAAAT-3' and reverse 5'-CGCTTC ACGAATTTGCGTGTCAT-3'; GAPDH, forward 5'-TGTTGC CATCAATGACCCCTT-3' and reverse 5'-CTCCACGACGTA CTCAGCG-3'; miR-421, forward 5'-CTCACTCACATCAAC AGACATTAATT-3' and reverse 5'-TATGGTTGTTCTGCT CTCTGTGTC-3'; and RIPK1, forward 5'-AGGCTTTGGGAA GGTGTCTC-3' and reverse 5'-CGGAGTACTCATCTCGGC TTT-3'. The thermocycling conditions were as follows: Initial denaturation at $95^{\circ} \mathrm{C}$ for $5 \mathrm{~min}$, followed by 40 cycles of denaturation at $95^{\circ} \mathrm{C}$ for $15 \mathrm{sec}$ and annealing/elongation at $60^{\circ} \mathrm{C}$ for $30 \mathrm{sec}$. The relative expression of genes was calculated by the $2^{-\Delta \Delta \mathrm{Cq}}$ method (27). Each experiment was performed in triplicate.

Western blotting assay. After treatment, cells were washed three times with ice-cold phosphate-buffered saline and then treated with RIPA lysis solution (Beijing Solarbio Science \& Technology Co., Ltd.) for $30 \mathrm{~min}$ to extract cellular proteins. Equal amounts (40 $\mu \mathrm{g} /$ lane) of protein were separated by $12 \%$ sodium dodecyl sulfate-polyacrylamide gel electrophoresis and then transferred to polyvinylidene fluoride membranes (EMD Millipore). The membranes were blocked with $5 \%$ skimmed milk in TBST containing $0.1 \%$ Tween at room temperature for $2 \mathrm{~h}$, followed by incubation with primary antibodies at $4^{\circ} \mathrm{C}$ overnight. Subsequently, the membranes were incubated with horseradish peroxidase (HRP)-conjugated secondary antibody at room temperature for $2 \mathrm{~h}$. The protein band was visualized using the enhanced chemiluminescence method using ECL reagent (EMD Millipore). GAPDH served as the loading control for normalization. The primary antibodies were as follows: Anti-protein kinase RNA-like endoplasmic reticulum kinase (PERK; cat no. 5683; 1:1,000), anti- $\alpha$ subunit of eukaryotic translation initiation factor 2 (eIF2 $\alpha$; cat no. 5324; 1:1,000), anti-C/EBP homologous protein (CHOP; cat no. 2895; 1:1,000), anti-RIPK1 (cat no. 3493; 1:1,000), anti-phosphorylated (p)-AKT (cat no. 4060; 1:1,000) and anti-p-mTOR (cat no. 5536; 1:1,000) and they were purchased from Cell Signaling Technology, Inc. Anti-p-PI3K was obtained from Biogot Technology, Co., Ltd. (cat no. BS4811; 1:1,000). The secondary antibodies were as follows: Anti-mouse IgG, HRP-conjugated antibody (cat no. 7076; 1:1,000) and anti-rabbit IgG, HRP-conjugated antibody (cat no. 7074; 1:1,000) were purchased from Cell Signaling Technology, Inc. Protein expression was quantified by performing AlphaView 3.4.0 software (ProteinSimple).

Flow cytometry analysis. Cell apoptosis detection was performed using the Annexin-V/propidium iodide (PI) Apoptosis Detection kit (Beyotime Institute of Biotechnology). Briefly, human melanocytes were plated into 6-well plates at a density of $2 \times 10^{5}$ cells per well. On the following day, the cells were transfected with inhibitor control, miR-421 inhibitor, miR-421 inhibitor + control-shRNA, or miR-421 inhibitor + RIPK1-shRNA. After transfection for $24 \mathrm{~h}$, the cells were treated with $3 \mu \mathrm{M}$ TM for $48 \mathrm{~h}$. Subsequently, the cells were 
collected, centrifuged with low temperature at high speed $\left(1,000 \mathrm{x} \mathrm{g} ; 5 \mathrm{~min} ; 4^{\circ} \mathrm{C}\right)$ and re-suspended in $100 \mu \mathrm{l}$ fluorescein isothiocyanate (FITC)-binding buffer. Subsequently, $\sim 5 \mu \mathrm{l}$ ready-to-use Annexin V-FITC (BD Bioscience) and $5 \mu \mathrm{l}$ PI were added to the buffer and the cells were incubated for $30 \mathrm{~min}$ at room temperature in the dark. Annexin V-FITC and PI fluorescence were assessed by BD FACSCalibur flow cytometer (BD Biosciences; Becton, Dickinson and Company). FlowJo software (version 7.6.1; FlowJo LLC) was used to analyze the data.

Dual-luciferase reporter assay. The bioinformatics software TargetScan 7.2 (http://www.targetscan.org/vert_72/) was used to predict the association between miR-421 and RIPK1, and the results demonstrated that miR-421 had binding sites for RIPK1. Subsequently, to confirm the binding sites between miR-421 and RIPK1, a dual-luciferase reporter assay was performed. In brief, luciferase reporter plasmids (psi-CHECK2) containing the wild-type as well as the mutant 3'UTRs of RIPK1, were manufactured by TsingKe Biotech. Human melanocytes were co-transfected with the wild-type or mutant 3'UTR luciferase reporter plasmids and the miR-421 mimic or the mimic control, respectively, using Lipofectamine 3000 (Invitrogen; Thermo Fisher Scientific, Inc.). Cells were harvested after transfection for $24 \mathrm{~h}$ and the luciferase activity was measured using the Dual-glo luciferase assay system (Promega Corporation) following the manufacturer's protocol. Firefly luciferase was used as a normalization control.

MTT assay. Cell viability was evaluated using the MTT assay (Beijing Solarbio Science \& Technology Co., Ltd.). Human melanocytes seeded in 96-well plates were treated according to the purpose of the experiment and $20 \mu \mathrm{l}$ MTT reagent was added into each well for another $4 \mathrm{~h}$ of incubation at $37^{\circ} \mathrm{C}$. Subsequently, $150 \mu \mathrm{l}$ dimethyl sulfoxide was added into each well and shaken for $15 \mathrm{~min}$. The optical density values were read at $490 \mathrm{~nm}$ using a microplate reader.

Statistical analysis. All data are presented as the mean \pm standard deviation. The SPSS 22.0 software (IBM Corp.) was used for statistical analysis and differences between groups were determined by Student's t-test or one way-analysis of variance followed by Tukey's post hoc test. $\mathrm{P}<0.05$ was considered to indicate a statistically significant difference.

\section{Results}

Expression of miR-421 in human melanocytes induced by ER stress. To evaluate the role of miR-421 in human melanocytes induced by ER stress, the levels of miR-421 expression were measured by RT-qPCR. Human melanocytes were treated with $3 \mu \mathrm{M}$ TM for $48 \mathrm{~h}$. A western blotting assay demonstrated that the expression of ER stress-related proteins, such as PERK, eIF2 $\alpha$ and CHOP, was upregulated (Fig. 1A), indicating that $3 \mu \mathrm{M} \mathrm{TM}$ activated ER stress in human melanocytes. MTT assay and flow cytometry indicated that TM significantly inhibited the viability $(\mathrm{P}<0.01$; Fig. 1B) and induced apoptosis $(\mathrm{P}<0.01$; Fig. $1 \mathrm{C}$ and $\mathrm{D})$ of human melanocytes. Moreover, an RT-qPCR assay was performed to detect the expression of miR-421 in human melanocytes. RT-qPCR analysis demonstrated that miR-421 expression was significantly upregulated in TM-induced human melanocytes compared with the control group $(\mathrm{P}<0.01$; Fig. 1E). Taken together, these findings confirmed that miR-421 expression was increased in human melanocytes induced by ER stress.

RIPKI is a direct target gene of miR-421. In order to study the underlying molecular mechanism, the TargetScan bioinformatic predication algorithms were used to predict the potential targets of miR-421. The results revealed the binding sites between RIPK1 and miR-421 (Fig. 2A). Subsequently, a dual-luciferase reporter assay was performed to confirm the binding sites between RIPK1 and miR-421. It was observed that the miR-421 mimic significantly increased the level of miR-421 in human melanocytes compared with the control group (Fig. 2B). miR-421 co-transfection with wild-type RIPK1 3'-UTR reporter inhibited the luciferase activity, but miR-421 exerted no effect on the reporter containing the mutant sequence (Fig. 2C). Therefore, RIPK1 was confirmed as a direct target gene of miR-421.

The expression of RIPK1 in TM-induced human melanocytes was next examined. Western blotting and the RT-qPCR assay demonstrated that, compared with the control group, TM significantly reduced the expression of RIPK1 in human melanocytes (Fig. 2D and E).

Effect of miR-421 inhibitor on the expression of ER stress-related proteins in TM-induced human melanocytes. Human melanocytes were transfected with the inhibitor control, miR-421 inhibitor, control-shRNA, RIPK1-shRNA or miR-421 inhibitor + RIPK1-shRNA for $24 \mathrm{~h}$ and then treated with TM $(3 \mu \mathrm{M})$ for $48 \mathrm{~h}$. The results indicated that, compared with the control group, the miR-421 inhibitor significantly reduced the expression of miR-421 in human melanocytes (Fig. 3A). Compared with the control group, RIPK1-shRNA significantly decreased the expression of RIPK1 in human melanocytes (Fig. 3B and C). In addition, RIPK1 expression was increased by the miR-421 inhibitor, which was obviously abolished by RIPK1-shRNA (Fig. 3D and E).

The expression of ER stress-related proteins was next examined. A western blotting assay revealed that, compared with the control group the protein expression of PERK, eIF2 $\alpha$ and CHOP was markedly increased in the TM treatment group; compared with the TM treatment group, the protein expression of PERK, eIF2 $\alpha$ and CHOP was obviously decreased in the $\mathrm{TM}+$ miR-421 inhibitor group, and this effect was eliminated by RIPK1-shRNA (Fig. 3F).

Effect of the miR-421 inhibitor on ER stress-induced human melanocyte damage. In order to investigate the effect of low expression of miR-421 on ER stress-induced damage of human melanocytes, an MTT assay and flow cytometry were performed. The results indicated that, compared with the control group, cell viability (Fig. 4A) was decreased and cell apoptosis (Fig. 4B and C) was increased in the TM treatment group. Compared with the TM treatment group, the miR-421 inhibitor significantly increased the viability of human melanocytes (Fig. 4A) and decreased cell apoptosis (Fig. 4B and C). All these changes were notably reversed by RIPK1-shRNA. 
A

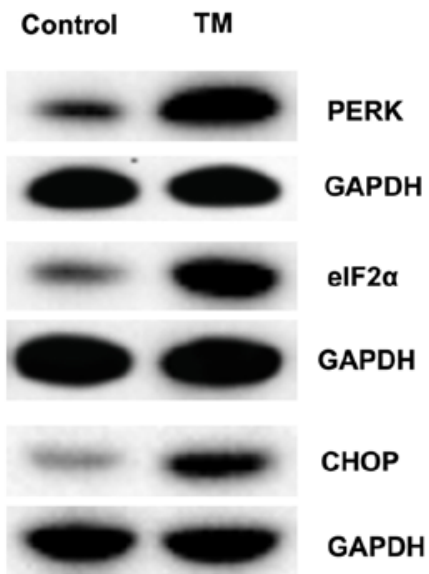

C
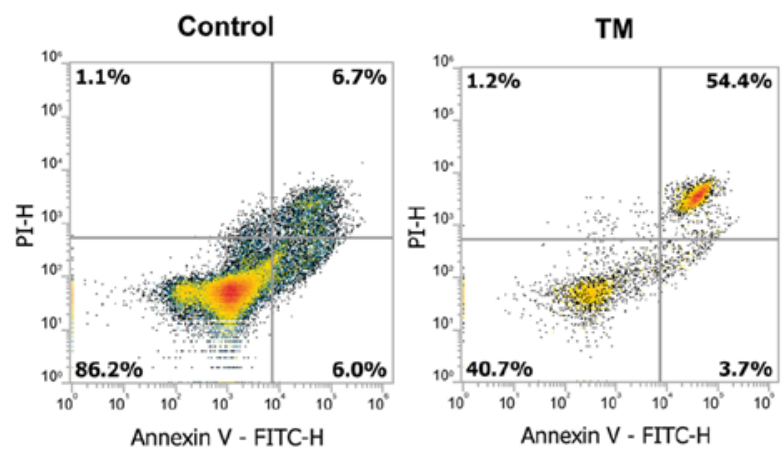

E

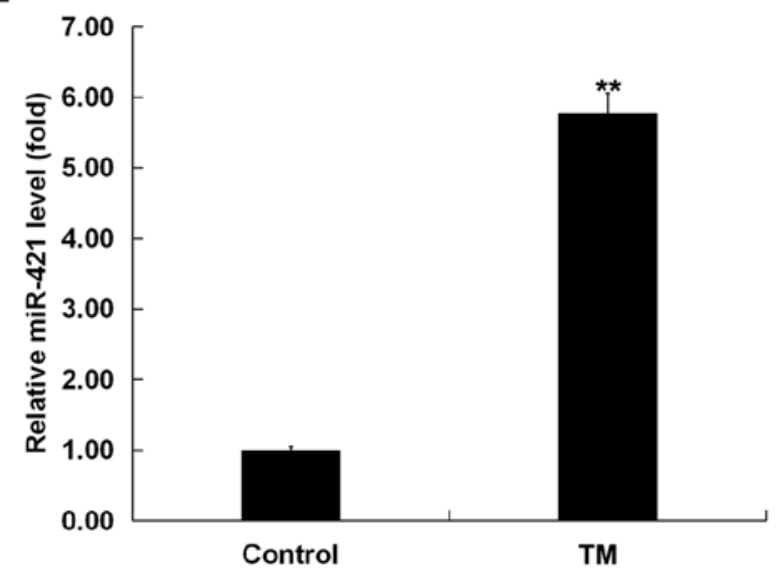

B

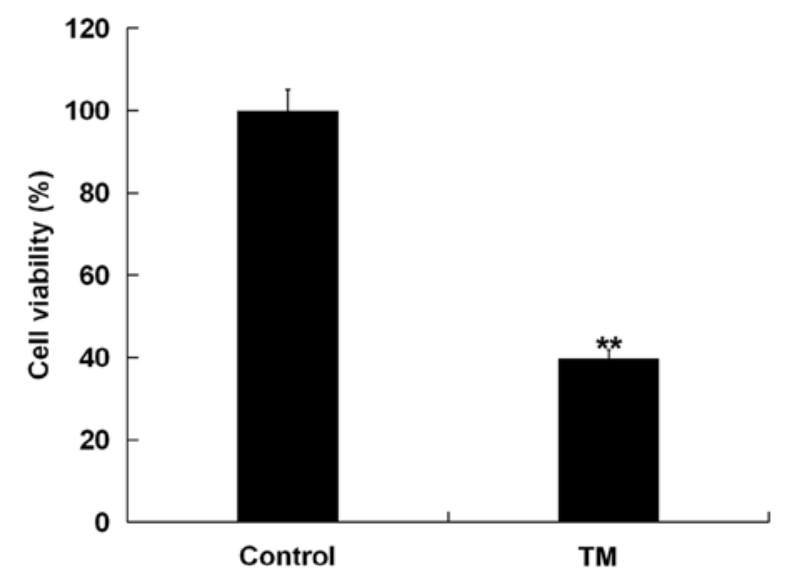

D

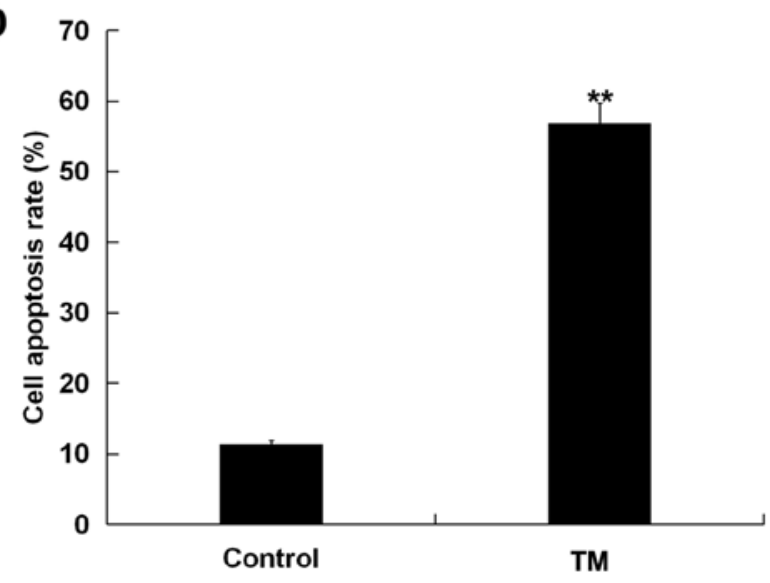

Figure 1. miR-421 is upregulated in human melanocytes induced by ER stress. (A) Western blot assay was used to estimate the protein expression of PERK, eIF2 $\alpha$ and CHOP in human melanocytes treated with $3 \mu \mathrm{M}$ TM for $48 \mathrm{~h}$. (B) MTT assay was performed to detect cell viability. (C) Flow cytometry and (D) analysis was performed to detect cell apoptosis. (E) Reverse transcription-quantitative PCR assay was performed to detect the expression of miR-421. Data are presented as the mean \pm standard deviation. ${ }^{* *} \mathrm{P}<0.01$ vs. the control. ER, endoplasmic reticulum; PERK, protein kinase RNA-like endoplasmic reticulum kinase; eIF2 $\alpha, \alpha$ subunit of eukaryotic translation initiation factor 2; CHOP, C/EBP homologous protein; FITC, fluorescein isothiocyanate; TM, tunicamycin; PI, propidium iodide; miR, microRNA.

Effect of the miR-421 inhibitor on the PI3K/AKT/mTOR signaling pathway in TM-induced human melanocytes. To investigate whether PI3K/AKT/mTOR signaling was involved in the effect of the miR-421 inhibitor on TM-induced human melanocytes, the expression of p-PI3K, PI3K, p-AKT, AKT, mTOR and p-mTOR in human melanocytes was examined. The western blot assay revealed that, compared with the control group, the protein expression of p-PI3K, PI3K, p-AKT,
AKT, mTOR and p-mTOR and the ratio of $\mathrm{p}-\mathrm{PI} 3 \mathrm{~K} / \mathrm{PI} 3 \mathrm{~K}$, $\mathrm{p}$-AKT/AKT and p-mTOR/mTOR were significantly decreased in the TM treatment group and, compared with the TM treatment group, the miR-421 inhibitor significantly increased the protein expression of p-PI3K, p-AKT and p-mTOR in the TM + miR-421 inhibitor group. The effect of the miR-421 inhibitor on TM-induced human melanocytes was markedly eliminated by RIPK1-shRNA (Fig. 5). Taken together, these findings 


\section{A}

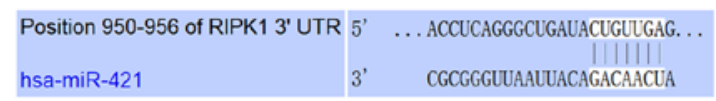

B

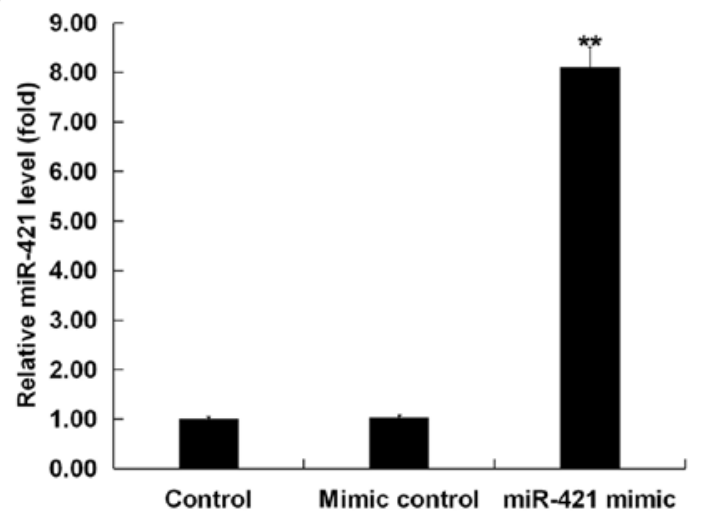

D

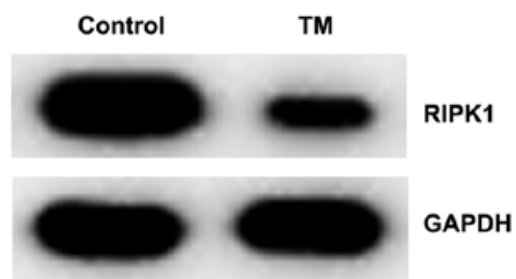

C

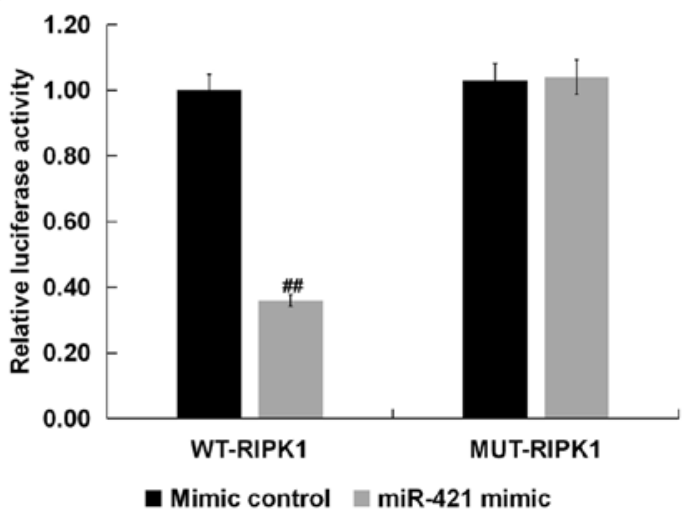

E

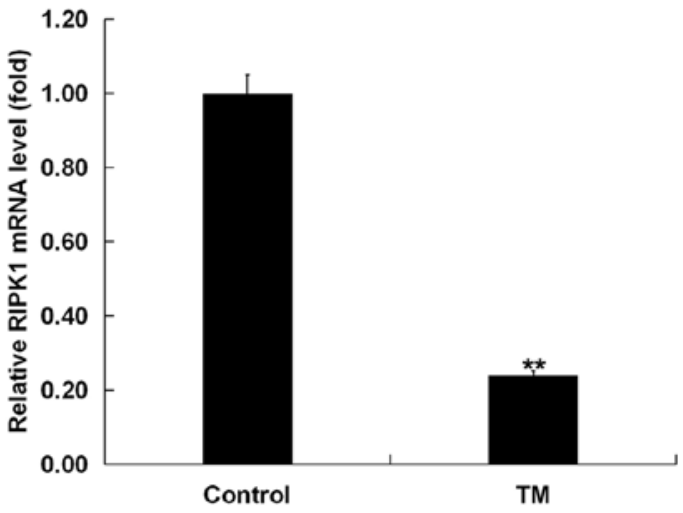

Figure 2. RIPK1 is a direct target gene of miR-421. (A) Prediction of miR-421 targeting RIPK1 by miRNA target gene database TargetScan. (B) RT-qPCR assay was used to detect the expression of miR-421. (C) Dual-luciferase reporter gene assay was used to verify the association between miR-421 and RIPK1. (D) Western blot and (E) RT-qPCR assays were used to measure the expression of RIPK1. Data were presented as the mean \pm standard deviation. ${ }^{* *} \mathrm{P}<0.01 \mathrm{vs.}$. the control; \# ${ }^{\#}<0.01$ vs. mimic control. RIPK1, receptor-interacting serine/threonine kinase 1; RT-qPCR, Reverse transcription-quantitative PCR; miR/miRNA, microRNA; TM, tunicamycin; MUT, mutant; WT, wild-type; UTR, untranslated.

indicate that miR-421 inhibitor activated the PI3K/AKT/mTOR signaling pathway in TM-induced human melanocytes.

\section{Discussion}

The present study demonstrated that the expression of the ER stress-related proteins PERK, eIF $2 \alpha$ and CHOP was upregulated in human melanocytes treated with $3 \mu \mathrm{M}$ TM for $48 \mathrm{~h}$. Moreover, TM inhibited human melanocyte viability and induced apoptosis. The results demonstrated that TM increased the expression of miR-421 in human melanocytes. To explore the role of miR-421, bioinformatics analysis was performed to predict its potential target genes. TargetScan predicted that RIPK1 was a potential target gene of miR-421. Dual-luciferase reporter gene assay further verified the association between miR-421 and RIPK1. The expression of RIPK1 was downregulated in human melanocytes treated with $3 \mu \mathrm{M}$ TM for $48 \mathrm{~h}$. Next, the effect of the downregulation of miR-421 on ER stress-induced damage of human melanocytes was investigated. miR-421 inhibitor reduced the expression of the ER stress-related proteins PERK, eIF2 $\alpha$ and CHOP in human melanocytes treated with TM. In addition, the miR-421 inhibitor promoted human melanocyte viability and decreased apoptosis, and these effects were reversed by RIPK1-shRNA. Finally, it was observed that the effect of the miR-421 inhibitor on human melanocytes was associated with the PI3K/AKT/mTOR signaling pathway.

Vitiligo is a non-continuous skin disease characterized by loss of pigment-producing skin cells (melanocytes), causing progressive skin depigmentation (28). At present, the treatment of vitiligo is mainly focused on preventing the development of the disease and achieving re-pigmentation of the non-pigmented area. Phototherapy is currently the preferred method of vitiligo treatment, but corticosteroids, surgery or local immunomodulators are also considered as treatment options for vitiligo (29-31). As a type of oxidative stress, ER stress is affected by adverse factors such as ischemia, hypoxia, hypoglycemia, drugs or poisons, which cause unfolded proteins to accumulate in the lumen of the ER. When the ability of the cell to repair is exceeded, the unfolded protein-mediated apoptosis may be induced. 
A
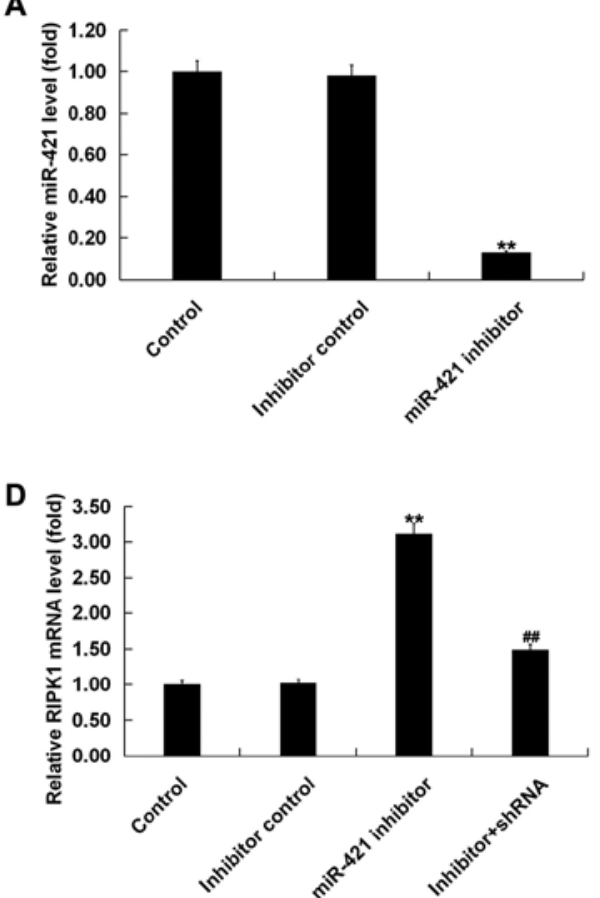

B

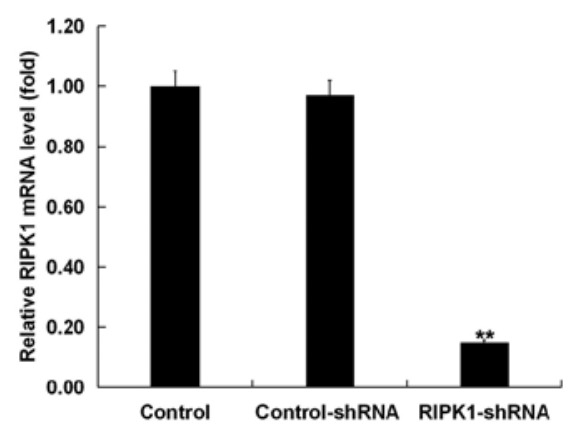

C

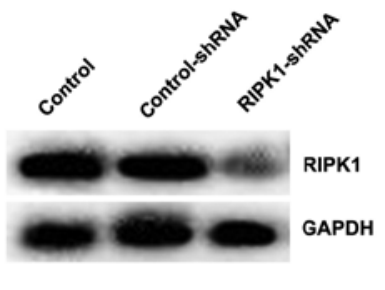

E

$\mathbf{F}$
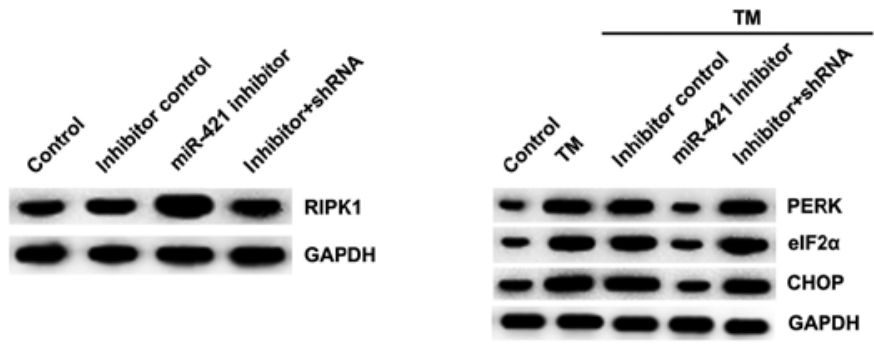

Figure 3. Effect of miR-421 inhibitor on the expression of ER stress-related proteins in TM-induced human melanocytes. (A) RT-qPCR analysis detected the expression of miR-421 in human melanocytes transfected with inhibitor control or miR-421 inhibitor for $24 \mathrm{~h}$. (B) RT-qPCR and (C) western blotting assays detected the expression of RIPK1 in human melanocytes transfected with control-shRNA or RIPK1-shRNA for $24 \mathrm{~h}$. (D) RT-qPCR assay and (E) western blotting assay were performed to detect the expression of RIPK1 in human melanocytes. (F) Western blot analysis of the protein expression of PERK, eIF2 $\alpha$ and $\mathrm{CHOP}$ in human melanocytes. Data were presented as the mean \pm standard deviation. ${ }^{* *} \mathrm{P}<0.01$ vs. the control; ${ }^{\# \#} \mathrm{P}<0.01$ vs. miR- 421 inhibitor. ER, endoplasmic reticulum; TM, tunicamycin; RIPK1, receptor-interacting serine/threonine kinase 1; PERK, protein kinase RNA-like endoplasmic reticulum kinase; eIF2 $\alpha, \alpha$ subunit of eukaryotic translation initiation factor 2; CHOP, C/EBP homologous protein; RT-qPCR, reverse transcription-quantitative PCR; miR, microRNA; sh, short hairpin.

A

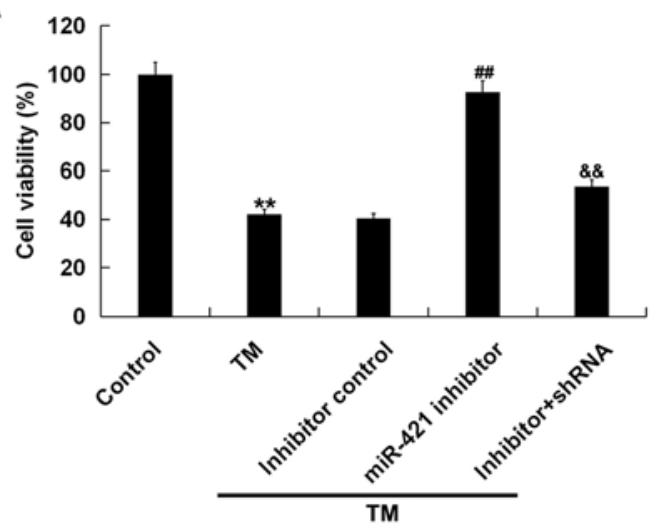

C

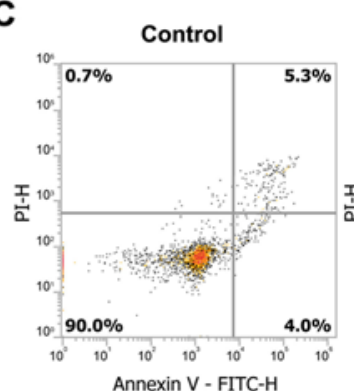

B

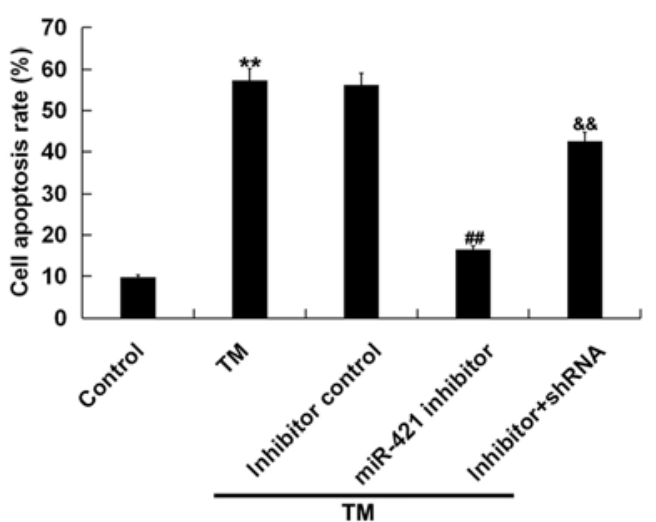

TM
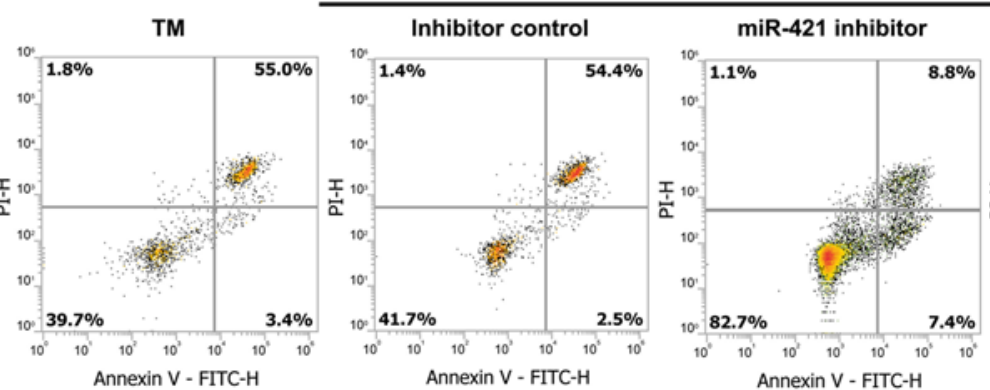

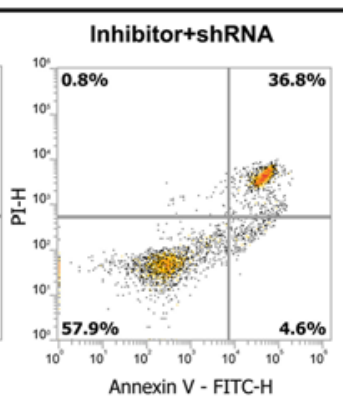

Figure 4. miR-421 inhibitor promotes viability and inhibits apoptosis in TM-induced human melanocytes. (A) MTT assay was performed to detect cell viability. (B) Flow cytometry and (C) analysis was performed to detect cell apoptosis. Data were presented as the mean \pm standard deviation. ${ }^{* *} \mathrm{P}<0.01$ vs. the control; ${ }^{\# /} \mathrm{P}<0.01$ vs. TM; ${ }^{\&} \mathrm{P}<0.01$ vs. TM + miR-421 inhibitor. TM, tunicamycin; miR, microRNA; sh, short hairpin; FITC, fluorescein isothiocyanate; PI, propidium iodide. 
A

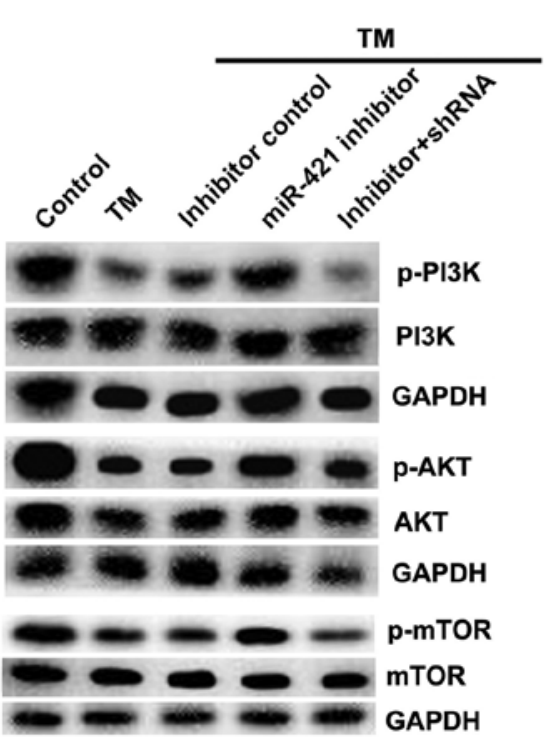

C

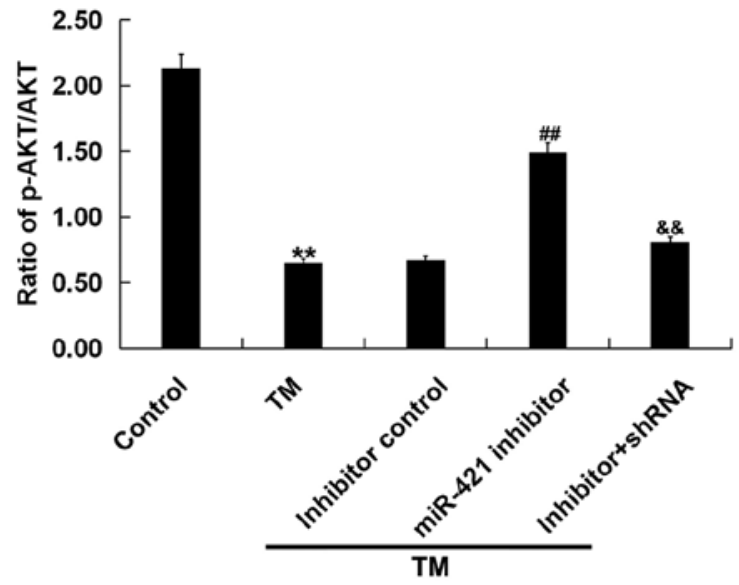

B

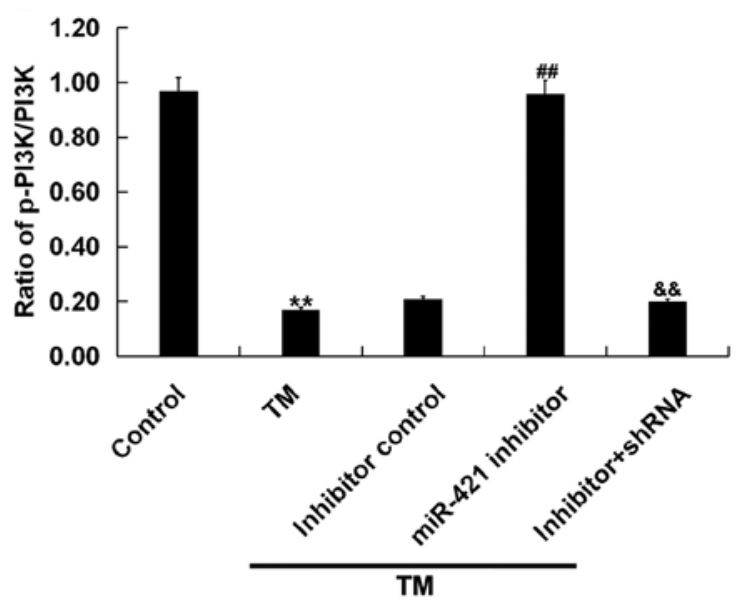

D

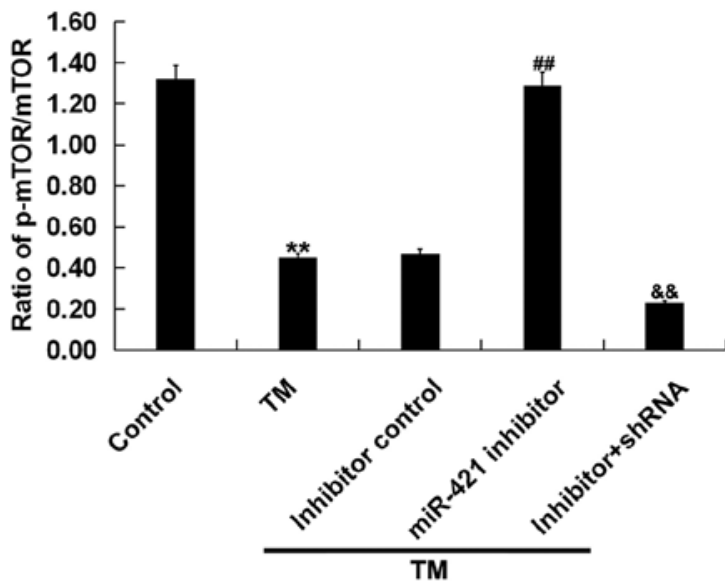

Figure 5. miR-421 inhibitor activates the PI3K/AKT/mTOR signaling pathway in TM-induced human melanocytes. (A) Western blot assay was performed to detect the protein expression of p-PI3K, PI3K, p-AKT, AKT, mTOR and p-mTOR in human melanocytes. The ratio of (B) p-PI3K/PI3K, (C) p-AKT/AKT and (D) $\mathrm{p}-\mathrm{mTOR} / \mathrm{mTOR}$ was calculated and presented. Data were presented as the mean \pm standard deviation. ${ }^{* *} \mathrm{P}<0.01$ vs. control; ${ }^{\# \#} \mathrm{P}<0.01$ vs. TM; ${ }^{\& \&} \mathrm{P}<0.01$ vs. TM + miR-421 inhibitor. TM, tunicamycin; PI3K, phosphoinositide3-kinase; p-AKT, phosphorylated-protein kinase B; mTOR, mammalian target of rapamycin.

It was previously demonstrated that miRNAs are involved in a number of key developmental pathways and different miRNAs may be associated with different diseases, such as inflammatory diseases, infections, developmental disorders and cancer (32). It has been reported that miR-211 plays a key role in the pathophysiology of human vitiligo and may be located at the apex of the normal melanocyte gene network (33). Shi et al (34) reported that overexpression of miR-25 promoted $\mathrm{H}_{2} \mathrm{O}_{2}$-induced melanocyte destruction and led to melanocyte dysfunction. miR-421, a specific miRNA, has been shown to promote the development of neuroblastoma through targeting the tumor suppressor Menin (35). Wu et al (16) indicated that miR-421 may regulate cell apoptosis via mediating caspase-3 in gastric cancer. However, to the best of our knowledge, there is currently no report on the role of miR-421 in vitiligo development. The results of the present study demonstrated that miR-421 expression was upregulated in human melanocytes induced by ER stress.
RIPK1 is a crucial regulator of TNFR1 signaling (17). It has been reported that RIPK1 is a target gene of miR-24-3p (36). In the present study, it was demonstrated by the dual-luciferase reporter assay that RIPK1 is a target of miR-421. Moreover, RIPK1 expression was downregulated in TM-induced human melanocytes.

ER stress activates the unfolded protein response pathways by inducing PERK, which increases the phosphorylation level of eIF2 $\alpha$ and the transcription activation of CHOP (37). Apoptosis mediators, such as PERK, CHOP and eIF2 $\alpha$, are involved in ER stress-associated cell death (38). These events indicate that ER stress activation of PERK, eIF2 $\alpha$ and CHOP protein expression plays a critical role in TM-induced human melanocyte apoptosis. Furthermore, PERK, eIF $2 \alpha$ and CHOP protein expression inhibition may inhibit ER stress-induced human melanocyte apoptosis, thus playing a protective role in vitiligo. As expected, the present study revealed that TM significantly increased the protein level of PERK, CHOP and 
eIF $2 \alpha$ in human melanocytes. The miR-421 inhibitor reduced the expression of the ER stress-related proteins PERK, eIF2 $\alpha$ and CHOP in human melanocytes treated with TM, and these effects were reversed by RIPK1-shRNA. Next, it was observed that low expression of miR-421 relieved ER stress-induced human melanocyte damage, but this effect was reversed by RIPK1-shRNA.

The PI3K/AKT/mTOR signaling pathway has been found to be associated with cell survival (21). Growth factors may protect against oxidative stress-induced apoptosis through activation of the AKT and mTOR pathways (22-24). AKT/mTOR complex activation suppresses ultraviolet-induced skin cell damage (39). PSORI-CM02, an empirically developed Chinese medicinal formula optimized from Yin Xie Ling, successfully treated psoriasis by inducing autophagy via inhibition of the PI3K/Akt/mTOR pathway (40). The $\mathrm{PI} 3 \mathrm{~K} / \mathrm{AKT} / \mathrm{mTOR}$ pathway has also been reported to play a critical role in atopic dermatitis (41). In addition, indirect data indicated that $\alpha$-MSH stimulated melanogenesis through the activation of the MEK/ERK or PI3K/AKT pathways (25). Modulation of the PI3K/AKT and mTOR pathway may be a novel approach to the clinical management of vitiligo (26). A previous study indicated that Nrf2 negatively regulates melanogenesis by modulating the PI3K/Akt signaling pathway (42). The $\alpha$-MSH-induced activation of the mTORC1 pathway, inhibited by rapamycin, helps to maintain dendrites of melanocytes under oxidative stress (26). Sustained PI3K activity can protect melanocytes from apoptosis, thereby indicating that the PI3K/AKT pathway plays a pivotal role in melanocyte survival (43). In the present study, it was investigated whether the miR-421 inhibitor affected the PI3K/AKT/mTOR signaling pathway in human melanocytes under ER stress and the results were the first to prove that ER stress inhibited the $\mathrm{PI} 3 \mathrm{~K} / \mathrm{AKT} / \mathrm{mTOR}$ signaling pathway in human melanocytes which was significantly activated by miR-421 inhibitor, while this effect was eliminated by RIPK1-shRNA.

In conclusion, the miR-421 inhibitor may protect human melanocytes from ER stress-induced cell damage through regulating $\mathrm{PI} 3 \mathrm{~K} / \mathrm{AKT} / \mathrm{mTOR}$ signaling and ER stress signaling by targeting RIPK1. The findings of the present study may provide a new therapeutic target and a theoretical basis for the treatment of vitiligo.

\section{Acknowledgements}

Not applicable.

\section{Funding}

The present study was supported by the National Natural Science Foundation of China (grant no. 81773335, 81803131 and 81602755), Zhejiang Provincial Natural Science Foundation (grant no. LY18H110001) and Zhejiang Basic Public Welfare Research Project (grant no. LGF18H110002).

\section{Availability of data and materials}

All data sets used and/or generated during the present study are available from the corresponding author on reasonable request.

\section{Authors' contributions}

XS and TW contributed to study design, data collection, statistical analysis, data interpretation and manuscript preparation. $\mathrm{BH}, \mathrm{GR}$ and AX contributed to data collection and statistical analysis.

\section{Ethics approval and consent to participate}

Not applicable.

\section{Patient consent for publication}

Not applicable.

\section{Competing interests}

The authors declare that they have no competing interests.

\section{References}

1. Ezzedine K, Eleftheriadou V, Whitton M and van Geel N: Vitiligo. Lancet 386: 74-84, 2015.

2. Jain A, Mal J, Mehndiratta V, Chander R and Patra SK: Study of oxidative stress in vitiligo. Indian J Clin Biochem 26: 78-81, 2010.

3. Silverberg JI and Silverberg NB: Association between vitiligo extent and distribution and quality-of-life impairment. JAMA Dermatol 149: 159-164, 2013.

4. Karelson M, Silm H and Kingo K: Quality of life and emotional state in vitiligo in an Estonian sample: Comparison with psoriasis and healthy controls. Acta Derm Venereol 93: 446-450, 2013

5. Jin Y, Birlea SA, Fain PR, Gowan K, Riccardi SL, Holland PJ, Mailloux CM, Sufit AJ, Hutton SM, Amadi-Myers A, et al: Variant of TYR and autoimmunity susceptibility loci in generalized vitiligo. New Engl J Med 362: 1686-1697, 2010.

6. Alghamdi KM, Khurrum H, Taieb A and Ezzedine K: Treatment of generalized vitiligo with anti-TNF- $\alpha$ agents. J Drugs Dermatol 11: 534-539, 2012.

7. Manga P, Elbuluk N and Orlow SJ: Recent advances in understanding vitiligo. F1000Res 5: pii: F1000 Faculty Rev-2234, 2016.

8. Eleftheriadou V, Whitton ME, Gawkrodger DJ, Batchelor J, Corne J, Lamb B, Ersser S, Ravenscroft J and Thomas KS: Future research into the treatment of vitiligo: Where should our priorities lie? Results of the vitiligo priority setting partnership. Br J Dermatol 164: 530-536, 2011.

9. Luan Q, Jin L, Jiang CC, Tay KH, Lai F, Liu XY, Liu YL, Guo ST, Li CY, Yan XG, et al: RIPK1 regulates survival of human melanoma cells upon endoplasmic reticulum stress through autophagy. Autophagy 11: 975-994, 2015.

10. Šahmatova L, Tankov S, Prans E, Aab A, Hermann H, Reemann P, Pihlap M, Karelson M, Abram K, Kisand K, et al: MicroRNA-155 is dysregulated in the skin of patients with vitiligo and inhibits melanogenesis-associated genes in melanocytes and keratinocytes. Acta Derm Venereol 96: 742-747, 2016.

11. Bijkerk R, de Bruin RG, van Solingen C, van Gils JM, Duijs JM, van der Veer EP, Rabelink TJ, Humphreys BD and van Zonneveld AJ: Silencing of microRNA-132 reduces renal fibrosis by selectively inhibiting myofibroblast proliferation. Kidney Int 89: 1268-1280, 2016.

12. Zu Y, Yang Y, Zhu J, Bo X, Hou S, Zhang B, Qiu J and Zheng J: MiR-146a suppresses hepatocellular carcinoma by downregulating TRAF6. Am J Cancer Res 6: 2502-2513, 2016.

13. Li Q, Zhang X, Li N, Liu Q and Chen D: miR-30b inhibits cancer cell growth, migration, and invasion by targeting homeobox A1 in esophageal cancer. Biochem Biophys Res Commun 485: 506-512, 2017.

14. Tian W, Wang G, Liu Y, Huang Z, Zhang C, Ning K, Yu C, Shen Y, Wang M, Li Y, et al: The miR-599 promotes non-small cell lung cancer cell invasion via SATB2. Biochem Biophys Res Commun 485: 35-40, 2017.

15. Hao J, Zhang S, Zhou Y, Liu C, Hu X and Shao C: MicroRNA 421 suppresses DPC4/Smad4 in pancreatic cancer. Biochem Biophys Res Commun 406: 552-557, 2011. 
16. Wu JH, Yao YL, Gu T, Wang ZY, Pu XY, Sun WW, Zhang X Jiang YB and Wang JJ: MiR-421 regulates apoptosis of BGC-823 gastric cancer cells by targeting caspase-3. Asian Pac J Cancer Prev 15: 5463-5468, 2014.

17. Pasparakis $M$ and Vandenabeele P: Necroptosis and its role in inflammation. Nature 517: 311-320, 2015.

18. Schneider AT, Gautheron J, Feoktistova M, Roderburg C, Loosen SH, Roy S, Benz F, Schemmer P, Büchler MW, Nachbur U, et al: RIPK1 Suppresses a TRAF2-dependent pathway to liver cancer. Cancer Cell 31: 94-109, 2017.

19. Saeed WK and Jun DW: Necroptosis: An emerging type of cell death in liver diseases. World J Gastroenterol 20: 12526-12532, 2014.

20. Shan B, Pan H, Najafov A and Yuan J: Necroptosis in development and diseases. Genes Dev 32: 327-340, 2018.

21. Cao $\mathrm{C}$ and Wan Y: Parameters of protection against ultraviolet radiation-induced skin cell damage. J Cell Physiol 220: 277-284, 2009.

22. Cao C, Huang X, Han Y, Wan Y, Birnbaumer L, Feng GS, Marshall J, Jiang M and Chu WM: Galpha(i1) and Galpha(i3) are required for epidermal growth factor-mediated activation of the Akt-mTORC1 pathway. Sci Signal 2: ra17, 2009.

23. Cao C, Lu S, Jiang Q, Wang WJ, Song X, Kivlin R, Wallin B, Bagdasarian A, Tamakloe T, Chu WM, et al: EGFR activation confers protections against UV-induced apoptosis in cultured mouse skin dendritic cells. Cell Signal 20: 1830-1838, 2008.

24. Cheng LB, Cheng L, Bi HE, Zhang ZQ, Yao J, Zhou XZ and Jiang Q: Alpha-melanocyte stimulating hormone protects retinal pigment epithelium cells from oxidative stress through activation of melanocortin 1 receptor-Akt-mTOR signaling. Biochem Biophys Res Commun 443: 447-452, 2014.

25. Kadekaro AL, Kavanagh R, Kanto H, Terzieva S, Hauser J, Kobayashi N, Schwemberger S, Cornelius J, Babcock G, Shertzer HG, et al: Alpha-Melanocortin and endothelin-1 activate antiapoptotic pathways and reduce DNA damage in human melanocytes. Cancer Res 65: 4292-4299, 2005.

26. Wan J, Lin F, Zhang W, Xu A, DeGiorgis J, Lu H and Wan Y: Novel approaches to vitiligo treatment via modulation of mTOR and NF-kB pathways in human skin melanocytes. Int J Biol Sci 13: 391-400, 2017

27. Livak KJ and Schmittgen TD: Analysis of relative gene expression data using real-time quantitative PCR and the 2(-Delta Delta C(T)) method. Methods 25: 402-408, 2001.

28. Le Poole IC, Das PK, van den Wijngaard RM, Bos JD and Westerhof W: Review of the etiopathomechanism of vitiligo: A convergence theory. Exp Dermatol 2: 145-153, 1993.

29. Gawkrodger DJ, Ormerod AD, Shaw L, Mauri-Sole I, Whitton ME, Watts MJ, Anstey AV, Ingham J and Young K: Vitiligo: Concise evidence based guidelines on diagnosis and management. Postgrad Med J 86: 466-471, 2010.

30. Gianfaldoni S, Wollina U, Tirant M, Tchernev G, Lotti J, Satolli F, Rovesti M, França K and Lotti T: Herbal compounds for the treatment of vitiligo: A review. Open Access Maced J Med Sci 6: 203-207, 2018.

31. Lotti T, Wollina U, Tchernev G, Valle Y, Lotti J, França K, Satolli F, Rovesti M, Tirant M, Lozev I, et al: An innovative therapeutic protocol for Vitiligo: Experience with the use of fraxel herbium laser, topical latanoprost and successive irradiation with UVA-1 Laser. Open Access Maced J Med Sci 6: 49-51, 2018.
32. Mehrgou A and Akouchekian M: Therapeutic impacts of microRNAs in breast cancer by their roles in regulating processes involved in this disease. J Res Med Sci 22: 130, 2017.

33. Spiegelman VS and Elcheva IA: Metabo-miR: miR-211 regulates mitochondrial energy metabolism in vitiligo. J Invest Dermatol 137: 1828-1830, 137.

34. Shi Q, Zhang W, Guo S, Jian Z, Li S, Li K, Ge R, Dai W, Wang G, Gao T and Li C: Oxidative stress-induced overexpression of miR-25: The mechanism underlying the degeneration of melanocytes in vitiligo. Cell Death Differ 23: 496-508, 2016.

35. Li Y, Li W, Zhang JG, Li HY and Li YM: Downregulation of tumor suppressor menin by miR-421 promotes proliferation and migration of neuroblastoma. Tumour Biol 35: 10011-10017, 2014.

36. Tan H, Qi J, Fan BY, Zhang J, Su FF and Wang HT: MicroRNA-24-3p attenuates myocardial ischemia/reperfusion injury by suppressing RIPK1 expression in mice. Cell Physiol Biochem 51: 46-62, 2018.

37. Walter $P$ and Ron $D$ : The unfolded protein response: From stress pathway to homeostaticregulation. Science 334: 1081-1086, 2011.

38. Li Y, Zhang Y, Fu H, Huang H, Lu Q, Qin H, Wu Y, Huang H, Mao G, Wei Z and Liao P: Hes1 knockdown exacerbates ischemic stroke following tMCAO by increasing ER stress-dependent apoptosis via the PERK/eIF2 $\alpha / \mathrm{ATF} 4 / \mathrm{CHOP}$ signaling pathway. Neurosci Bull, Jul 15, 2019 (Epub ahead of print).

39. Umeda J, Sano S, Kogawa K, Motoyama N, Yoshikawa K, Itami $\mathrm{S}$, Kondoh $\mathrm{G}$, Watanabe $\mathrm{T}$ and Takeda J: In vivo cooperation between $\mathrm{Bcl}-\mathrm{xL}$ and the phosphoinositide 3-kinase-Akt signalingpathway for the protection of epidermal keratinocytes from apoptosis. FASEB J 17: 610-620, 2003.

40. Yue L, Ailin W, Jinwei Z, Leng L, Jianan W, Li L, Haiming C, Ling $\mathrm{H}$ and Chuanjian L: PSORI-CM02 ameliorates psoriasis in vivo and in vitro by inducing autophagy via inhibition of the PI3K/Akt/mTOR pathway. Phytomedicine 64: 153054, 2019.

41. Arshad Z, Rezapour-Firouzi S, Mohammadian M and Ebrahimifar: The sources of essential fatty acids for allergic and cancer patients; a connection with insight into mammalian target of rapamycin: A narrative review. Asian Pac J Cancer Prev 19: 2391-2401, 2018

42. Shin JM, Kim MY, Sohn KC, Jung SY, Lee HE, Lim JW, Kim S, Lee YH, Im M, Seo YJ, et al: Nrf2 negatively regulates melanogenesis by modulating PI3K/Akt signaling. PLoS One 9: e96035, 2014.

43. Larribere L, Khaled M, Tartare-Deckert S, Busca R, Luciano F, Bille K, Valony G, Eychene A, Auberger P, Ortonne JP, et al: PI3K mediates protection against TRAIL-induced apoptosis in primary human melanocytes. Cell Death Differ 11: 1084-1091, 2004.

This work is licensed under a Creative Commons Attribution-NonCommercial-NoDerivatives 4.0 International (CC BY-NC-ND 4.0) License. 